\title{
Solution Processing and Self-Organization of PbS Quantum Dots Passivated with Formamidinium Lead lodide $\left(\mathrm{FAPbl}_{3}\right)$
}

Samaneh Aynehband, Maryam Mohammadi, Kerstin Thorwarth, Roland Hany, Frank Alain Nüesch,* Marta D. Rossell, Robin Pauer, Jean-Michel Nunzi, and Abdolreza Simchi*

Cite This: ACS Omega 2020, 5, 15746-15754

Read Online

\section{ACCESS | Llll Metrics \& More | 回 Article Recommendations | (s) Supporting Information}

ABSTRACT: Solution-processed lead sulfide quantum dots (PbS QDs) are very attractive as NIR-active semiconductors for the fabrication of cost-efficient optoelectronic devices. To control the thin film carrier transport, as well as stability, surface passivation is of crucial importance. Here, we present the successful surface passivation of $\mathrm{PbS}$ QDs by the formamidinium lead iodide $\left(\mathrm{FAPbI}_{3}\right)$ ligand. $\mathrm{An}$ effective procedure for the fabrication of $\mathrm{FAPbI}_{3}$-passivated $\mathrm{PbS}$ QDs through a binary-phase ligand exchange protocol in hexane and $n$-methylformamide is demonstrated. It is shown that this solution-processed ligand exchange drastically changes the photoluminescence intensity, exciton recombination dynamics, and carrier lifetime of the nanocrystals. The solution casting of the ligand-exchanged nanocrystals into thin films results in the periodic ordering of QDs in a square superlattice with close contacts. Planar graphene/QD photodetectors fabricated with $\mathrm{PbS}$ QDs passivated with $\mathrm{FAPbI}_{3}$ show substantially increased thermal stability as

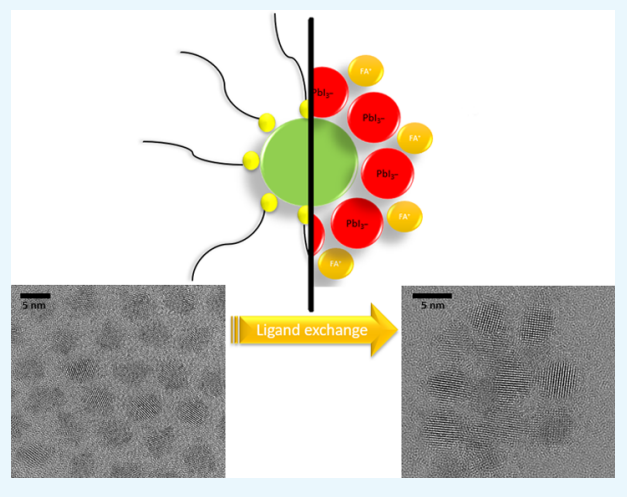
compared to similar devices using $\mathrm{PbS}$ QDs passivated with commonly used methylammonium lead iodide.

\section{INTRODUCTION}

During the past decade, band-gap tunability, simple and lowcost synthesis, high charge carrier mobility, and special optoelectronic properties of semiconductor quantum dots (QDs) have attracted tremendous interest for solutionprocessed optoelectronic devices. ${ }^{1}$ Of particular interest, monodispersed $\mathrm{PbS}$ QDs can easily be processed from earthabundant materials, exhibiting size-tuned band gaps over a broad spectral range extending to the near-infrared. ${ }^{2,3}$ Owing to their useful properties, particularly large Bohr radius (20 $\mathrm{nm}$ ) and high charge carrier mobility rendered possible by short conjugated ligands, applications of $\mathrm{PbS} \mathrm{QDs}$ in optoelectronic devices have surged up. ${ }^{4-6}$ Due to the high surface-to-volume ratio of $\mathrm{PbS} \mathrm{QDs}$, the electronic properties are highly affected by the surface characteristics because the surface states dictate the position and the number of states near the band gap. ${ }^{7}$ Accordingly, using these QDs in optoelectronic devices requires surface treatments to improve the electronic coupling and electron transport between QDs. ${ }^{8-10}$ To enhance the stability of QDs, long ligands are commonly used for surface passivation. However, the steric hindrance prevents long organic ligands from penetrating the intercation trenches on the surface of QDs, resulting in incomplete surface passivation. Unpassivated surface sites are susceptible to attack by oxygen and/or water, leading to instability of the QDs in air. ${ }^{11}$ Furthermore, surface passivation by short ligands is not only more effective but also improves the conductivity of $\mathrm{QD}$ films. In fact, the charge carrier mobility in QD thin films is enhanced by several orders of magnitude through conjugating with short ligands. Therefore, complete surface passivation by short ligands is necessary to make them stable and to prevent their degradation. Various approaches and different types of ligands have been employed for the surface treatment of QDs to form highly conductive arrays and films. ${ }^{1,12-17}$ Solution and solid-state ligand exchange methods have widely been used. ${ }^{1}$ The former works are based on the transferring of long alkyl-capped $\mathrm{PbS}$ QDs (e.g., oleic acid (OA)-PbS) from a nonpolar solvent to a polar solvent through ligand exchange with shorter capping molecules (e.g., mercaptopropionic acid, MPA, or 2ethanedithiol). In the latter procedure, the washing of deposited films with an organic solution containing short ligands is performed for the ligand exchange. The great advantages of the former over the latter method are the possibility of ink-printing as the one-step film fabrication process and complete passivation of the QD surfaces. ${ }^{1,18}$

All-inorganic perovskite structures in the form of $\mathrm{CsPbX}_{3}(\mathrm{X}$ $=\mathrm{I}, \mathrm{Br}, \mathrm{Cl}$ ) have a similar crystal structure and low lattice mismatch with $\mathrm{PbS}^{19}$ Organic-inorganic perovskites, for

Received: May 18, 2020

Accepted: June 5, 2020

Published: June 19, 2020 




Figure 1. (a) Schematic presentation and photographic images of colloidal PbS QDs processed by the solution ligand exchange protocol. The effect of ligand exchange treatment on (b) absorption, (c) steady-state PL, (d) time decay PL, and (e) FTIR spectrum of PbS QDs. PL and FTIR were measured in colloidal hexane and $\mathrm{N}$-methylformamide solutions for $\mathrm{OA}$ and $\mathrm{FAPbI}_{3}$, respectively. Absorption spectra were measured on spincoated films.

example, methylammonium lead halides $\left(\mathrm{CH}_{3} \mathrm{NH}_{3} \mathrm{PbX}_{3}\right.$ or $\mathrm{MAPbX}_{3}$ ), are intensively used in thin film solar cells because of their superior properties like high charge carrier diffusion lengths, high charge carrier mobility, and broad absorption range. Another interesting point is their perfect lattice matching with $\mathrm{PbS}$ QDs. ${ }^{20}$ Recently, $\mathrm{CH}_{3} \mathrm{NH}_{3} \mathrm{PbX}_{3}$ perovskites have successfully been employed as stable capping ligands for a variety of QDs like $\mathrm{PbS}, \mathrm{CdS}, \mathrm{InP}$, and $\mathrm{CdSe}{ }^{21}$ As a result, efficient electronic passivation and highly luminescent QDs have been attained. The solid-state ligand exchange method has also been employed for the replacement of oleate and oleylamine to achieve $\mathrm{PbS}-\mathrm{CH}_{3} \mathrm{NH}_{3} \mathrm{PbX}_{3} .{ }^{20}$ As compared to $\mathrm{PbS}$ QDs, $\mathrm{PbS}-\mathrm{CH}_{3} \mathrm{NH}_{3} \mathrm{PbI}_{3}$ QDs exhibit complementary optical absorption spectra, facile charge separation, high conductivities, and efficient charge transport. Choi et al. ${ }^{22}$ prepared a high-quality $\mathrm{PbS} \mathrm{QD}$ ink through the solution ligand exchange process by the use of $\mathrm{MAPbI}_{3}$ as the capping material. The $\mathrm{PbS}$ nanocrystals were stabilized by $\mathrm{PbI}_{3}$ anions and MA cations as an electrical double layer. By using this ink, they attained solar cells with a power conversion efficiency of $3.7 \%$.

To the best of our knowledge, $\mathrm{MAPbX}_{3}$ are the only organic-inorganic capping materials, which have been used so far to enhance the absorption region and improve the electron transport of PbS QD films. Unfortunately, the weak stability of methylammonium lead iodide ${ }^{23,24}$ is limiting their optoelectronic applications. To improve the stability of the perovskite, formamidinium (FA) was used to replace methylammonium (MA) cations. $^{25}$ Formamidinium lead halide perovskites $\left(\mathrm{CH}\left(\mathrm{NH}_{2}\right)_{2} \mathrm{PbX}_{3}\right.$ or $\left.\mathrm{FAPbX}_{3}\right)$ have recently been considered as a promising alternative for the thermodynamically less stable MA perovskites. The FA cation is larger (1.9-2.2 $\AA$ ) than the MA cation (1.8 $\AA$ ), thus narrowing the band-gap energy of FAPbI ${ }_{3}{ }^{26}$ The higher stability of the $\mathrm{FAPbI}_{3}$ structure is attributed to the higher number of hydrogen bonds between the hydrogen atoms of the ammonium cations and iodine ions in the inorganic cage. ${ }^{27}$ 
In this paper, we introduce $\mathrm{FAPbI}_{3}$ as a more effective organic-inorganic ligand for the surface passivation of $\mathrm{PbS}$ QDs. Drastic changes in the photoluminescence (PL) properties and exciton recombination dynamics of $\mathrm{PbS}$ QDs are demonstrated. The lifetime of charge carriers is significantly reduced as compared with that of OA-capped $\mathrm{PbS}$ QDs. In addition, the formation of nanocrystal superlattices during film formation is shown. Also, by using $\mathrm{PbS}-\mathrm{FAPbI}_{3} \mathrm{QDs}$ in photodetectors, we obtain an improvement in both response amplitude and thermal stability with respect to a similar device where the active layer consists of $\mathrm{PbS}-\mathrm{MAPbI}_{3}$.

\section{RESULTS AND DISCUSSION}

A schematic illustration of the solution-processed ligand exchange via $\mathrm{FAPbI}_{3}$ is shown in Figure 1a. We also observed an increased solubility of $\mathrm{PbS}-\mathrm{FAPbI}_{3}$ as compared to that of $\mathrm{PbS}-\mathrm{MAPbI}_{3}$ as precipitation of the suspended particles in NMF by centrifugation at $14000 \mathrm{rpm}$ was much harder in the case of $\mathrm{PbS}-\mathrm{FAPbI}_{3}$ (see SI, Figure S2). The absorption spectra of the $\mathrm{PbS} \mathrm{QD}$ solution before and after ligand exchange with $\mathrm{FAPbI}_{3}$ are illustrated in Figure $1 \mathrm{~b}$. Two distinct absorption peaks in the NIR region are visible. The main absorption peaks before the ligand exchange are located at 914 and $1292 \mathrm{~nm}$. These peaks shifted to about 894 and $1390 \mathrm{~nm}$ after the treatment. Therefore, the exchanged QDs absorb a broader range of the spectrum from visible to NIR, which makes them more suitable for photodetector devices.

The PL spectra in the solution of $\mathrm{OA}-\mathrm{PbS} \mathrm{QDs}$ and after ligand exchange with $\mathrm{FAPbI}_{3}$ are shown in Figure 1c. The emission peaks are located at $1350 \mathrm{~nm}$ for OA and $1480 \mathrm{~nm}$ for FA. Such large red shifts upon replacing oleate ligands by iodide salts have been reported and attributed to the decrease in wavefunction confinement due to a shallower HOMO level of the adsorbed iodide. ${ }^{28}$ Additionally, a change in the absorption peak position can occur upon aggregation when using short capping ligands. ${ }^{29}$ This is accompanied by a drastic change in the luminescence quantum efficiency and a muchreduced exciton lifetime (typically $1 \mathrm{~ns}$ ) due to defect state quenching. Although we do observe a reduced quantum efficiency and lifetime, the values obtained in this work are higher than for the bare aggregated PbS QDs. Red shifts in the excitonic absorption and emission peaks can also be induced via interparticle coupling between the QDs. ${ }^{29-31}$ Such coupling leads to both a significant red shift and a reduction in the quantum efficiency of luminescence via exciton dissociation due to charge carrier tunneling from dot to dot. While this process is expected in solid films, it is less expected to occur in solution. In the case of large QD size distributions, however, the unexpected large Stokes shifts of up to $200 \mathrm{meV}$ could be attributed to aggregation in solution. ${ }^{32}$ It is noteworthy that the Stokes shift in our case is around 50 $\mathrm{meV}$. We therefore rather exclude the possibility of aggregation. Observation of a blue shift from 914 to $894 \mathrm{~nm}$ in the first absorption peak after ligand exchange can be ascribed to the formation of minibands induced by electron coupling between adjacent nanocrystals. ${ }^{33,34}$ The formation of the minibands is very important for efficient carrier collection in optoelectronic devices. ${ }^{33,34}$

The ligand exchange treatment not only induced a red shift in the emission peak but also significantly quenched the PL intensity. At the excitation wavelength of $565 \mathrm{~nm}$, PLQY values of 15 and $1.5 \%$ were determined before and after ligand exchange, respectively. The result of time-resolved PL (TRPL) spectroscopy is shown in Figure 1d. We employed a biexponential model to fit the experimental data, ${ }^{29}$ and the extracted parameters are compiled in Table 1.

Table 1. TRPL Fitting Results for PbS QDs Passivated with $\mathrm{OA}$ and $\mathrm{FAPbI}_{3}$ Ligands

\begin{tabular}{lcccc}
\multicolumn{1}{c}{ sample } & $A_{1}$ & $\tau_{1}(\mathrm{~ns})$ & $A_{2}$ & $\tau_{2}(\mathrm{~ns})$ \\
$\mathrm{PbS}-\mathrm{OA}$ & 0.88 & 1076 & 0.88 & 1076 \\
$\mathrm{PbS}-\mathrm{FAPbI}_{3}$ & 0.41 & 10.3 & 0.20 & 1777 \\
\hline
\end{tabular}

$$
I(t)=A_{0}+A_{1} \exp \left(-t / \tau_{1}\right)+A_{2} \exp \left(-t / \tau_{2}\right)
$$

$\tau_{1}$ and $\tau_{2}$ show the fast and slow decay components, where $A_{1}$ and $A_{2}$ represent their amplitudes. The results display that the ligand exchange treatment reduces the exciton lifetime and changes the relaxation mechanisms. A long organic ligand can interrupt the charge transfer between QDs and lead to the slow radiative recombination process. Formamidinium lead iodide decreases the interparticle distances between QDs so that the nonradiative recombination becomes prominent. ${ }^{29,35}$

Figure 1e depicts the FTIR spectra of PbS QDs before and after ligand exchange. Four characteristic peaks of OA are observed at 2925, 2852, 1542, and $1400 \mathrm{~cm}^{-1}$. ${ }^{36}$ These peaks disappear after the ligand exchange treatment, which indicates removing of OA upon the exchange process. The peaks of $\mathrm{N}-$ $\mathrm{H}$ and $\mathrm{C}=\mathrm{N}$ bonds are visible at 3359 and $1712 \mathrm{~cm}^{-1}$, respectively. ${ }^{37,38}$ This observation indicates the existence of the perovskite phase on the surface of $\mathrm{PbS} \mathrm{QDs}$. Apart from these peaks, all other peaks are related to the solvent.

Figure 2 exhibits the full survey and high-resolution XPS spectra of $\mathrm{PbS}-\mathrm{OA}$ and $\mathrm{PbS}-\mathrm{FAPbI}_{3}$ QD films that were spincast on gold-coated substrates. Table 2 lists the atomic composition of the samples for the relevant elements. The XPS analysis corroborates the successful exchange of ligands. Evidence comes from the fact that the oxygen signal in $\mathrm{PbS}-\mathrm{FAPbI}_{3} \mathrm{QD}$ is missing, while it is clearly present in the $\mathrm{PbS}-\mathrm{OA}$ sample, confirming the presence of oleic acid (Figure $2 \mathrm{~d}$ ). Albeit the carbon/oxygen ratio of 11.8 is somewhat higher than its theoretical value of 9 , the relative atomic concentration confirms the presence of oleic acid since carbon contamination from the atmosphere during sample transfer and mounting cannot be excluded. Further evidence for the ligand exchange comes from the presence of iodide for the $\mathrm{PbS}-\mathrm{FAPbI}_{3} \mathrm{QD}$ samples (Figure 2c). From Table 2, we infer an iodide/lead ratio of 0.61 , which is substantially lower than the value of 3 expected for $\mathrm{PbI}_{3}^{-}$. Also, in the case of $\mathrm{PbS}-\mathrm{FAPbI}_{3} \mathrm{QD}$ samples, we observe double peak features for the $\mathrm{Pb} 4 \mathrm{f}_{7 / 2}$ and $\mathrm{Pb} 4 \mathrm{f}_{5 / 2}$ peaks with a separation energy of $1 \mathrm{eV}$, respectively (Figure 2e). This separation energy corresponds well to the chemical shift observed between the $\mathrm{Pb} 4 \mathrm{f}_{7 / 2}$ peak of $\mathrm{PbS}$ $(137.7 \mathrm{eV})^{39}$ and $\mathrm{FAPbI}_{3}(138.6 \mathrm{eV}) .^{40}$ Transmission electron microscopy (TEM) analysis indicates that the $\mathrm{FAPbI}_{3}$ ligand is thinner than $1 \mathrm{~nm}$ (Figure 4), and therefore a strong lead signal from the $\mathrm{PbS}$ core is indeed expected. An alternative interpretation of the shoulder on the low-energy side of the $\mathrm{Pb}$ $4 \mathrm{f}_{7 / 2}$ peak has previously been attributed to the metallic $\mathrm{Pb}^{0}$ present on organo-metal halide-capped $\mathrm{PbS}$ nanoparticles after ligand exchange. ${ }^{22}$ However, the expected chemical shift between $\mathrm{FAPbI}_{3}$ and $\mathrm{Pb}^{0}(136.9 \mathrm{eV})^{41}$ would be $1.7 \mathrm{eV}$, which is significantly larger than the peak-to-peak separation of $1.0 \mathrm{eV}$ observed here. Another argument against the assign- 



Figure 2. Survey spectrum of passivated $\mathrm{PbS}$ QDs with (a) oleic acid and (b) FAPbI ${ }_{3}$. High-resolution XPS spectra of (c) I 3d $\mathrm{d}^{5}$, (d) O 1s, (e) Pb 4f, and (f) $\mathrm{C} 1 \mathrm{~s}$.

Table 2. Concentration of Components (atom \%) Determined by XPS

\begin{tabular}{lllll}
\multicolumn{1}{c}{ sample } & $\mathrm{C} 1 \mathrm{~s}$ & $\mathrm{O} 1 \mathrm{~s}$ & ${\mathrm{I} 3 \mathrm{~d}^{5}}$ & $\mathrm{~Pb} 4 \mathrm{f}$ \\
$\mathrm{PbS}-\mathrm{OA}$ & 69.75 & 5.93 & 0 & 8.36 \\
$\mathrm{PbS}-\mathrm{FAPbI}_{3}$ & 25.08 & 0.56 & 5.01 & 8.17 \\
\hline
\end{tabular}

ment to metallic lead is the very low $\mathrm{I} / \mathrm{Pb}=0.6$ ratio, meaning that the concentration of $\mathrm{Pb}^{0}$ would be about twice that of $\mathrm{PbI}_{3}^{-}$. This would contradict the very similar intensity we observe for the double $\mathrm{Pb} 4 \mathrm{f}_{7 / 2}$ peak. We note that there is a considerable shift of the $\mathrm{PbS}-\mathrm{FAPbI}_{3} \mathrm{XPS}$ peaks to lower binding energies. This could arise from charging effects and from the interface dipole induced by the ligand. ${ }^{42}$ An in-depth investigation of these effects, however, is beyond the scope of this work.

The crystallinity of QDs was assessed by electron diffraction (ED). The ED pattern in Figure 3a shows well-defined diffraction rings corresponding to the rock-salt structure of $\mathrm{PbS}$. The simulated diffraction rings calculated using the $F m \overline{3} m$ space group and lattice parameter $a=0.5934 \mathrm{~nm}^{43}$ confirm an excellent agreement with the experimental data. Figure $3 b$ shows a high-resolution transmission electron microscopy (HRTEM) image of a PbS nanocrystal oriented with its [001] zone axis perpendicular to the image plane. HRTEM studies showed that monodispersed nanocrystals with an average size of $4.7 \pm 0.2 \mathrm{~nm}$ were synthesized. The nanoparticles have a truncated octahedron or rhombicuboctahedron shape with (100)-, (110)-, and (111)-type facets. The Fourier transform (FT) of the nanocrystal (Figure 3c) and a line profile extracted along the [100] direction (marked with a red rectangle in Figure $3 \mathrm{~d}$ ) confirm the lead sulfide rock-salt structure.

Overview zero-loss filtered TEM images of the films prepared from $\mathrm{PbS}-\mathrm{OA}$ and $\mathrm{PbS}-\mathrm{FAPbI}_{3} \mathrm{QDs}$ are shown in Figure 4. In Figure 4a, large domains of QDs organized in a hexagonal close-packed fashion are visible. Figure $4 \mathrm{~b}$ reveals much smaller domains of QDs organized in a square-packed fashion. FT patterns extracted from the selected areas (indicated by the red squares) are shown as insets. The FT spots reveal the self-organization of the nanocrystals during film formation. The reciprocal distances were used to extract the superlattice spacing. The FT pattern shown in Figure 4a reveals that the $\mathrm{PbS}$ QDs are organized in a hexagonal closepacked fashion and are spaced by $0.18 \mathrm{~nm}^{-1}$, i.e., $5.6 \mathrm{~nm}$. The FT image presented in Figure $4 \mathrm{~b}$ shows that, at the studied region of the film, the nanocrystals are organized in a squarepacked fashion and are separated by $0.21 \mathrm{~nm}^{-1}$, i.e., $4.8 \mathrm{~nm}$. HRTEM studies at higher magnifications reveal that the nanocrystals organized in a hexagonal close-packed fashion are not fused together but separated about $1 \mathrm{~nm}$ (Figure 4c). In contrast, the exchanged QDs arranged in a square-packed fashion often appear to have necking points along the (100)type facets (Figure $4 \mathrm{~d}$ ), in line with the previous reports. ${ }^{44}$ 

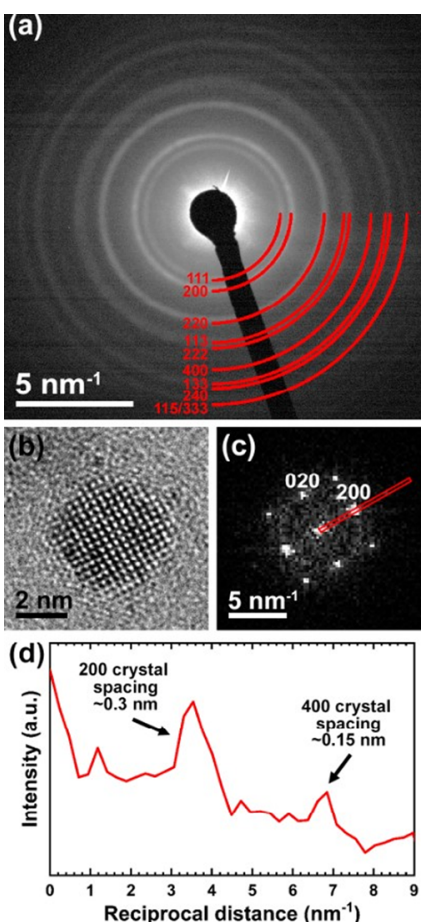

Figure 3. (a) ED pattern of $\mathrm{PbS}-\mathrm{OA}$ QDs with overlaid simulated diffraction rings. (b) HRTEM image of a PbS $\mathrm{QD}$ and (c) corresponding FT pattern. (d) Line profile obtained along the [100] direction of the FT in panel (c) (indicated by the red rectangle).
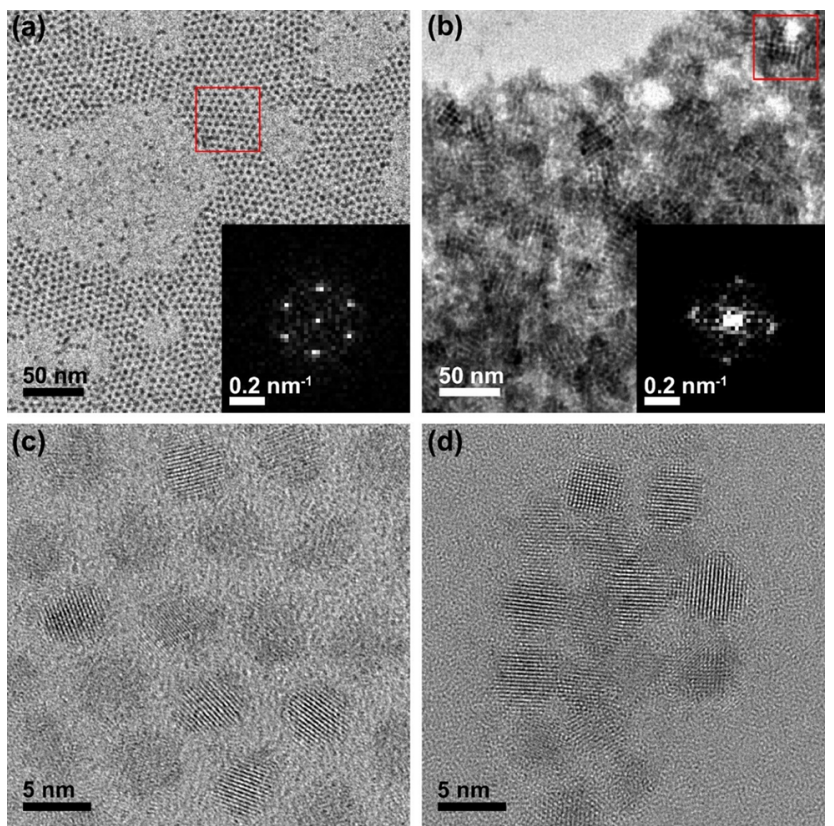

Figure 4. Low-magnification zero-loss filtered TEM images of $\mathrm{PbS}$ QDs capped with (a) OA and (b) $\mathrm{FAPbI}_{3}$. The insets are FT patterns extracted from the areas indicated by the red squares. Selforganization of QDs in hexagonal- and square-packed fashions is visible. HRTEM images of (c) PbS-OA and (d) $\mathrm{PbS}-\mathrm{FAPbI}_{3}$ QDs.

These results reveal a reduction of the interparticle spacing of the nanocrystals in the thin film after the ligand exchange.

As the $\mathrm{PbS}$ nanocrystals present a truncated octahedron or rhombicuboctahedron shape, three different facets, including (100), (110), and (111) orientations, are exposed to the ligands. The (100)-type facets are the least acidic ones having a lead coordination number of $5 .^{45}$ The (110) and (111) facets have lead coordination numbers of 4 and 3, respectively, with a higher affinity toward anionic ligands. ${ }^{44}$ However, due to the lower surface energy of the 100-type facets as compared to that of the (111)-type ones, ligands can more easily be removed from (100). ${ }^{44}$ During the ligand exchange, anionic perovskite ligands prefer (110) and (111) facets, while neighboring QDs are connected along the 100-type facets resulting in the squarepacked nanocrystals. ${ }^{21,44}$

To assess the stability of the different QD ligands, we chose the planar graphene/QD photodetector architecture (SI, Figure S1), since the latter is less sensitive to the morphology of the QD film. Figure 5a,b shows the current response of the graphene/QD photodetectors at an applied source-drain voltage of $3 \mathrm{~V}$ during alternating irradiation $(\mathrm{ON})$ and dark (OFF) periods. Devices using QDs with a $\mathrm{MAPbI}_{3}$ ligand and QDs with a $\mathrm{FAPbI}_{3}$ ligand show photocurrents of $0.6-0.7 \mu \mathrm{A}$, respectively. The mechanism of photoconduction in channels with graphene/ $\mathrm{PbS}$ quantum dot heterostructures has been elucidated. ${ }^{46}$ Upon irradiation, electron-hole pairs are induced in the $\mathrm{PbS}$ QDs, which can both be transferred to graphene with an extremely high carrier mobility of up to $200000 \mathrm{~cm}^{2} /$ $\mathrm{Vs}^{46}$ given the fact that the Fermi level of graphene lies in the band gap of $\mathrm{PbS}$. However, the rates of electron and hole transfers differ, which induces a net electronic charge on the QDs and a compensating positive charge on graphene. By this photodoping effect, the conductivity of graphene is changed, which gives rise to the observed photocurrent response (see Figure $5 \mathrm{c}) .{ }^{46}$ This principle was shown to lead to high optical gain and enhanced optical response. ${ }^{47}$ Numerous studies in the literature indicate that $\mathrm{MAPbI}_{3}$ is thermodynamically unstable with respect to decomposition forming lead iodide, hydroiodic acid, and methylamine gas in the absence of air. ${ }^{48}$ Heat accelerates this process as can be clearly seen in the shortened lifetime of solar cell devices. ${ }^{48}$ Therefore, perovskite layers with increased stability have been developed by replacing MA by the larger organic cation FA. Here, we have investigated whether the stability of perovskite ligands on $\mathrm{PbS}$ QDs also follows this trend. For this purpose, we have investigated the aging of thin film graphene/QD photodiode layers at $130{ }^{\circ} \mathrm{C}$ for $1 \mathrm{~h}$ (Figure $5 \mathrm{~b}$ ). While the photocurrent decrease in the case of FA was only $4 \%$, the decrease in the case of MA was $40 \%$ and therefore significantly higher. We attribute the deterioration of the devices upon annealing to the accumulation of decomposition products at the graphene interface, reducing the photoinduced charge transfer and due to the agglomeration of QDs, reducing the absorption coefficient of the latter. ${ }^{49}$

\section{CONCLUSIONS}

In summary, we have employed a facile and solution-based procedure at room temperature to exchange long alkyl ligands (such as $\mathrm{OA}$ ) with an organic-inorganic ligand $\left(\mathrm{FAPbI}_{3}\right.$ perovskite) for $\mathrm{PbS} \mathrm{QDs}$. High-resolution transmission electron microscopy characterizations revealed self-organization of the nanocrystals in superlattices during thin film formation. The ligand-exchanged QDs organized in squarepacked lattices instead of hexagonal-packed structures with shorter interparticle distances. The ligand exchange produced a red shift in the absorption and emission spectrum and promoted additional exciton-quenching channels. Also, the $\mathrm{FAPbI}_{3}$ ligand showed superior thermal stability in graphene/ 

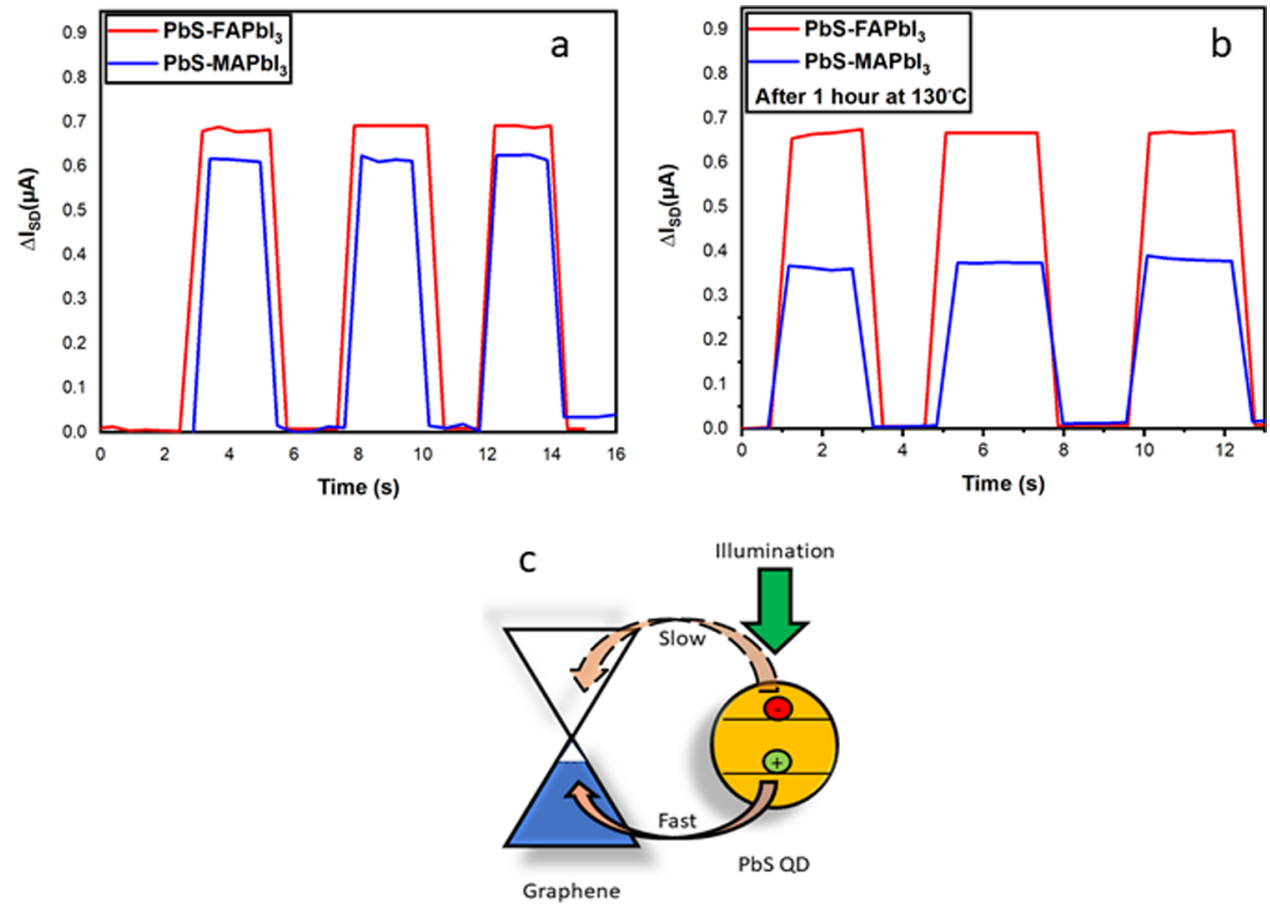

Figure 5. Current response of graphene $/ \mathrm{PbS}-\mathrm{FAPbI}_{3}$ and $\mathrm{PbS}-\mathrm{MAPbI}{ }_{3} \mathrm{QD}$ phototransistors under white light illumination (a) before and (b) after maintaining at $130{ }^{\circ} \mathrm{C}$ for $1 \mathrm{~h}$, and (c) schematic illustration of the photodoping effect in graphene/PbS QDs.

QD photodetectors as compared to similar devices using QDs using the $\mathrm{MAPbI}_{3}$ ligand. The proposed approach for the passivation of $\mathrm{PbS}$ QDs with a formamidinium-based organicinorganic perovskite may pave a new way for the development of optoelectronic devices.

\section{EXPERIMENTAL SECTION}

Materials. Lead(II) acetate trihydrate $\left(\mathrm{Pb}\left(\mathrm{CH}_{3} \mathrm{COO}\right)_{2}\right.$. $3 \mathrm{H}_{2} \mathrm{O}$, 99.99\%, Sigma-Aldrich), bis(trimethylsilyl)sulfide $\left(\mathrm{TMS}_{2} \mathrm{~S}\right.$, Sigma-Aldrich), lead(II) iodide $\left(\mathrm{PbI}_{2}, 99 \%\right.$, SigmaAldrich), formamidinium iodide (FAI, 99\%, Sigma-Aldrich), 1octadecene (ODE, 90\%, Sigma-Aldrich), oleic acid (OA, 90\%, Sigma-Aldrich), $\mathrm{N}$-methylformamide (NMF, $\mathrm{HCONHCH}_{3}$, 99\%, Sigma-Aldrich), ethanol (Fluka), hexane (95\%, SigmaAldrich), acetone (99.5\%, Sigma-Aldrich), titanium(IV) isopropoxide (TTIP, 97\%, Sigma-Aldrich), hydrochloric acid (HCL, Sigma-Aldrich), and methylammonium iodide (MAI, $98 \%$, Sigma-Aldrich) were used without further purification. $\mathrm{Si} / \mathrm{SiO}_{2} /$ graphene substrates (Graphenea, Spain) were utilized for device fabrication.

Synthesis of PbS QDs. The synthesis of PbS QDs was performed by using a hot-injection method according to the protocol reported by Hines and co-workers. ${ }^{2}$ Briefly, $1.5 \mathrm{~g}$ $\mathrm{Pb}\left(\mathrm{CH}_{3} \mathrm{COO}\right)_{3} \cdot 3 \mathrm{H}_{2} \mathrm{O}, 35 \mathrm{~mL}$ 1-octadecene (ODE), and 15 $\mathrm{mL} O A$ were mixed in a three-neck flask. The resulting solution was degassed under vacuum for $2 \mathrm{~h}$ at $120{ }^{\circ} \mathrm{C}$ followed by stirring at $143{ }^{\circ} \mathrm{C}$ under a nitrogen atmosphere. In another container, $0.42 \mathrm{~mL} \mathrm{TMS}_{2} \mathrm{~S}$ was mixed with $10 \mathrm{~mL}$ of dried ODE. The second solution was injected into the first one at $143{ }^{\circ} \mathrm{C}$. The growth time was $3 \mathrm{~min}$. The reaction mixture was then cooled to room temperature. The washing process was performed with hexane/ethanol (solvent/nonsolvent) three times, redissolved in hexane, and finally filtered through a $0.2 \mu \mathrm{m}$ PTFE filter.
Solution Ligand Exchange. The method developed by Dirin and co-workers ${ }^{21}$ was utilized for the ligand exchange in the solution phase. Formamidinium (methylammonium) iodide $(50 \mu \mathrm{mol})$ and $50 \mu \mathrm{mol}$ of lead iodide were dissolved in NMF. This ligand solution was mixed with $5 \mathrm{mg}$ PbS QDs in $1 \mathrm{~mL}$ hexane. The biphasic system was stirred for $4.5 \mathrm{~h}(1 \mathrm{~h})$ until complete migration of QDs occurs. Afterward, the nonpolar phase was removed, and the nanocrystals were washed three times with $1 \mathrm{~mL}$ hexane. The FA-passivated QDs were then precipitated by adding an appropriate amount of acetone followed by dispersion in NMF. For comparison, $\mathrm{PbS}$ QDs were also passivated with $\mathrm{MAPbI}_{3}$ ligands according to a

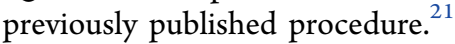

Device Fabrication. The purchased $\mathrm{Si} / \mathrm{SiO}_{2} /$ graphene substrate $\left(\mathrm{SiO}_{2}\right.$ thickness of $300 \mathrm{~nm}$ coated with a graphene monolayer) was cut to $2 \times 2 \mathrm{~cm}^{2}$ pieces inside the glovebox (see SI, Figure S1a). Thereafter, interdigitated source and drain gold electrodes with a channel length of $250 \mu \mathrm{m}$ and a width of $5 \mathrm{~mm}$ were deposited by physical vapor deposition (PVD). To compare the effect of the perovskite ligands, $10 \mu \mathrm{L}$ of $\mathrm{QD}$ solutions (before and after ligand exchange with $\mathrm{MAPbI}_{3}$ or $\mathrm{FAPbI}_{3}$ ) were deposited on graphene via dropcasting $(80 \mathrm{~nm})$. Then, the samples were placed on a hot plate at $70{ }^{\circ} \mathrm{C}$ for $10 \mathrm{~min}$ to dry the active layer. After fabrication, the devices were either directly characterized or placed in an oven at $130{ }^{\circ} \mathrm{C}$ for $1 \mathrm{~h}$ to test their thermal stability. The device architecture and a photograph of the devices are given in the SI (Figure S1).

\section{CHARACTERIZATIONS}

Microscopy. The size and morphology of the nanocrystals were observed by a JEOL 2200FS TEM equipped with an incolumn Omega-type energy filter. The instrument was operated at $200 \mathrm{keV}$ and used for electron diffraction (ED), HRTEM, and energy-filtered transmission electron microscopy 
(EFTEM). A multifunctional field-emission scanning electron microscope (NanoSEM 230) was employed for observation of the prepared thin films.

Spectroscopy. The absorption spectrum of the assynthesized and ligand-exchanged colloidal $\mathrm{PbS} \mathrm{QDs}$ was recorded on a Shimadzu UV-3600 UV-vis-IR spectrophotometer. Steady-state and time-resolved PL measurements were conducted on a Fluorolog Horiba Jobin Yvon and a NanoLog FL3 Horiba spectrofluorimeter, respectively. A Vector Bruker 22/DigilabBioRad FTS 6000 FT-IR Spectrometer was used for the FTIR measurement. XPS spectra were acquired on a Physical Electronics (PHI) Quantum 2000 Scanning ESCA Microprobe System using monochromated Al $\mathrm{K} \alpha$ radiation $(h \nu=1486.7 \mathrm{eV})$ and a hemispherical capacitor electron-energy analyzer equipped with a channel plate and a position-sensitive detector. The electron take-off angle was $45^{\circ}$, and the analyzer was operated in the constant pass energy mode at $23.5 \mathrm{eV}$ for the detailed spectra of the $\mathrm{Cu} 2 \mathrm{p}_{3 / 2}, \mathrm{O} 1 \mathrm{~s}$, $\mathrm{C} 1 \mathrm{~s}$, and $\mathrm{N} 1 \mathrm{~s}$ peaks, and a step size of $0.20 \mathrm{eV}$ was used. The beam diameter was typically $150 \mu \mathrm{m}$. The binding energy is calibrated using $\mathrm{Cu} 2 \mathrm{p}_{3 / 2}, \mathrm{Ag} 3 \mathrm{~d}_{5 / 2}$, and $\mathrm{Au} 4 \mathrm{f}_{7 / 2}$ at 932.62, 368.21 , and $83.96 \mathrm{eV}$, respectively, to within $\pm 0.1 \mathrm{eV}$ (see ISO.15472; 2010-05). To compensate for possible surface charging, built-in electron and argon ion neutralizers were used. The base pressure of the system was below $5 \times 10^{-7} \mathrm{~Pa}$. The spectra were analyzed using the software MultiPak 8.2B, and the peaks are shown after a Shirley background subtraction. The atomic concentrations were calculated using the corrected relative sensitivity factors, as given by the manufacturer, and normalized to 100 atom \%. The relative uncertainty is around $10 \%$.

Device Performance. Current versus time curves were measured by a Keithley 4200 SCS parametric analyzer, equipped with a SuperK compact supercontinuum white pulsed laser unit (NKT Photonics, 450-2400 nm range). The pulse width of the laser source was $2 \mathrm{~ns}$ at a repetition rate of 1 $\mathrm{kHz}$ with a total output power of $110 \mathrm{~mW}$. Devices were alternatively measured in the dark and under laser irradiation for time periods of several seconds each. A constant bias $V_{\mathrm{SD}}$ of $3 \mathrm{~V}$ was applied to the source contact during the photocurrent transients and the source-gate voltage was set to $0 \mathrm{~V}$.

\section{ASSOCIATED CONTENT}

\section{SI Supporting Information}

The Supporting Information is available free of charge at https://pubs.acs.org/doi/10.1021/acsomega.0c02319.

Electronic image of the phototransistor and the schematic illustration of the device architecture; and optical images and the corresponding absorption curves of the supernatant taken after different times of centrifugation (PDF)

\section{AUTHOR INFORMATION}

\section{Corresponding Authors}

Frank Alain Nüesch - Laboratory for Functional Polymers, Empa, Swiss Federal Laboratories for Materials Science and Technology, 8600 Dübendorf, Switzerland; Institute of Materials Science and Engineering, EPFL, Ecole Polytechnique Féderale de Lausanne, 1015 Lausanne, Switzerland; ำ orcid.org/0000-0003-0145-7611; Email: Frank.Nueesch@ empa.ch
Abdolreza Simchi - Department of Materials Science and Engineering and Institute for Nanoscience and Nanotechnology, Sharif University of Technology, 14588 Tehran, Iran; ○ orcid.org/0000-0002-9111-2977; Email: simchi@ sharif.edu

\section{Authors}

Samaneh Aynehband - Department of Materials Science and Engineering, Sharif University of Technology, 14588 Tehran, Iran; Laboratory for Functional Polymers, Empa, Swiss Federal Laboratories for Materials Science and Technology, 8600 Dübendorf, Switzerland; Department of Chemistry, Department of Physics, Engineering Physics and Astronomy, Queens University, Kingston, Ontario K7L 3N6, Canada

Maryam Mohammadi - Department of Materials Science and Engineering, Sharif University of Technology, 14588 Tehran, Iran

Kerstin Thorwarth - Surface Science and Coating Technologies, Empa, Swiss Federal Laboratories for Materials Science and Technology, 8600 Dübendorf, Switzerland

Roland Hany - Laboratory for Functional Polymers, Empa, Swiss Federal Laboratories for Materials Science and Technology, 8600 Dübendorf, Switzerland; 다이.org/00000003-0569-119X

Marta D. Rossell - Electron Microscopy Center, Empa, Swiss Federal Laboratories for Materials Science and Technology, 8600 Dübendorf, Switzerland

Robin Pauer - Electron Microscopy Center, Empa, Swiss Federal Laboratories for Materials Science and Technology, 8600 Dübendorf, Switzerland

Jean-Michel Nunzi - Department of Chemistry, Department of Physics, Engineering Physics and Astronomy, Queens University, Kingston, Ontario K7L 3N6, Canada; Orcid.org/00000001-5490-4273

Complete contact information is available at:

https://pubs.acs.org/10.1021/acsomega.0c02319

\section{Notes}

The authors declare no competing financial interest.

\section{ACKNOWLEDGMENTS}

The contribution of Dr Ulrich Müller (EMPA) through scientific discussions in XPS results is highly appreciated. The authors also thank Dr Maksym Kovalenko, Dr Dmitry Dirin, and Dr Sergii Yakunin (ETHZ University) for their expertise and assistance throughout synthesis and TRPL measurement of quantum dots. The authors acknowledge the Sharif University of Technology (Grant No. QA970816), the Iran National Science Foundation (INSF No. 95-S-48740), and the Natural Sciences and Engineering Research Council of Canada, Discovery Grants program (RGPIN-2015-05485).

\section{REFERENCES}

(1) Wang, R.; Shang, Y.; Kanjanaboos, P.; Zhou, W.; Ning, Z.; Sargent, E. H. Colloidal quantum dot ligand engineering for high performance solar cells. Energy Environ. Sci. 2016, 9, 1130-1143.

(2) Hines, M.; Scholes, G. Colloidal PbS Nanocrystals with SizeTunable Near-Infrared Emission: Observation of Post-Synthesis SelfNarrowing of the Particle Size Distribution. Adv. Mater. 2003, 15, 1844-1849.

(3) McDonald, S. A.; Konstantatos, G.; Zhang, S.; Cyr, P.; Klem, E.; Levina, L.; Sargent, E. Solution-processed PbS quantum dot infrared photodetectors and photovoltaics. Nat. Mater. 2005, 4, 138-142. 
(4) Yu, D.; Wang, C.; Guyot-Sionnest, P. n-Type Conducting CdSe Nanocrystal Solids. Science 2003, 300, 1277-1280.

(5) Hetsch, F.; Zhao, N.; Kershaw, S.; Rogach, A. Quantum Dot Field Effect Transistors. Mater. Today 2013, 16, 312-325.

(6) Tavakoli, M. M.; Aashuri, H.; Simchi, A.; Kalytchuk, S.; Fan, Z. Quasi Core/Shell Lead Sulfide/Graphene Quantum Dots for Bulk Heterojunction Solar Cells. J. Phys. Chem. C 2015, 119, 1888618895.

(7) Li, Y.; Zhu, J.; Huang, Y.; Wei, J.; Liu, F.; Shao, Z.; Hu, L.; Chen, S.; Yang, S.; Tang, J.; Yao, J.; Dai, S. Efficient inorganic solid solar cells composed of perovskite and $\mathrm{PbS}$ quantum dots. Nanoscale 2015, 7, 9902-9907.

(8) Padilha, L. A.; Stewart, J. T.; Sandberg, R. L.; Bae, W. K.; Koh, W.-K.; Pietryga, J. M.; Klimov, V. I. Carrier Multiplication in Semiconductor Nanocrystals: Influence of Size, Shape, and Composition. Acc. Chem. Res. 2013, 46, 1261-1269.

(9) Böhm, M. L.; Jellicoe, T. C.; Tabachnyk, M.; Davis, N. J. L. K.; Wisnivesky-Rocca-Rivarola, F.; Ducati, C.; Ehrler, B.; Bakulin, A. A.; Greenham, N. C. Lead Telluride Quantum Dot Solar Cells Displaying External Quantum Efficiencies Exceeding 120\%. Nano Lett. 2015, 15, 7987-7993.

(10) Semonin, O.; Luther, J.; Choi, S.; Chen, H.-Y.; Gao, J.; Nozik, A.; Beard, M. Peak External Photocurrent Quantum Efficiency Exceeding $100 \%$ via MEG in a Quantum Dot Solar Cell. Science 2011, 334, 1530-1533.

(11) Yuan, M.; Liu, M.; Sargent, E. Colloidal Quantum Dot Solids for Solution-Processed Solar Cells. Nat. Energy 2016, 1, 1601616025 .

(12) Tayyebi, A.; Tavakoli, M. M.; Outokesh, M.; Shafiekhani, A.; Simchi, A. A. Supercritical Synthesis and Characterization of Graphene-PbS Quantum Dots Composite with Enhanced Photovoltaic Properties. Ind. Eng. Chem. Res. 2015, 54, 7382-7392.

(13) Tavakoli, M. M.; Mirfasih, M. H.; Hasanzadeh, S.; Aashuri, H.; Simchi, A. Surface passivation of lead sulfide nanocrystals with low electron affinity metals: photoluminescence and photovoltaic performance. Phys. Chem. Chem. Phys. 2016, 18, 12086-12092.

(14) Shestha, A.; Yin, Y.; Andersson, G. G.; Spooner, N. A.; Qiao, S.; Dai, S. Versatile PbS Quantum Dot Ligand Exchange Systems in the Presence of Pb-Thiolates. Small 2017, 13, No. 1602956.

(15) Tavakoli, M. M.; Tayyebi, A.; Simchi, A.; Aashuri, H.; Outokesh, M.; Fan, Z. Physicochemical properties of hybrid graphene-lead sulfide quantum dots prepared by supercritical ethanol. J. Nanopart. Res. 2015, 17, 1-13.

(16) Gu, M.; et al. Stable PbS quantum dot ink for efficient solar cells by solution-phase ligand engineering. J. Mater. Chem. A 2019, 7, 15951-15959.

(17) Abargues, R.; Navarro, J.; Rodríguez-Cantó, P. J.; Maulu, A.; Sánchez-Royo, J. F.; Martínez-Pastor, J. P. Enhancing the photocatalytic properties of $\mathrm{PbS} \mathrm{QD}$ solids: the ligand exchange approach. Nanoscale 2019, 11, 1978-1987.

(18) Ning, Z.; et al. Air-stable n-type colloidal quantum dot solids. Nat. Mater. 2014, 13, 822-828.

(19) Zhang, X.; Zhang, J.; Phuyal, D.; Du, J.; Tian, L.; Öberg, V. A.; Johansson, M. B.; Cappel, U. B.; Karis, O.; Liu, J.; Rensmo, H.; Boschloo, G.; Johansson, E. M. J. Inorganic CsPbI3 Perovskite Coating on $\mathrm{PbS}$ Quantum Dot for Highly Efficient and Stable Infrared Light Converting Solar Cells. Adv. Energy Mater. 2018, 8, No. 1702049

(20) Peng, J.; Chen, Y.; Zhang, X.; Dong, A.; Liang, Z. Solid-State Ligand-Exchange Fabrication of $\mathrm{CH} 3 \mathrm{NH} 3 \mathrm{PbI} 3$ Capped $\mathrm{PbS}$ Quantum Dot Solar Cells. Adv. Sci. 2016, 3, No. 1500432.

(21) Dirin, D. N.; Dreyfuss, S.; Bodnarchuk, M. I.; Nedelcu, G.; Papagiorgis, P.; Itskos, G.; Kovalenko, M. V. Lead Halide Perovskites and Other Metal Halide Complexes As Inorganic Capping Ligands for Colloidal Nanocrystals. J. Am. Chem. Soc. 2014, 136, 6550-6553.

(22) Choi, H.; Lee, J.-G.; Xuan Dung, M.; Beard, M.; Yoon, S.; Jeong, S. Supersonically Spray-Coated Colloidal Quantum Dot Ink Solar Cells. Sci. Rep. 2017, 7, No. 622.
(23) Fan, Y.; Qin, H.; Ye, W.; Liu, M.; Huang, F.; Zhong, D. Improving the stability of methylammonium lead iodide perovskite solar cells by cesium doping. Thin Solid Films 2018, 667, 40-47.

(24) Brunetti, B.; Cavallo, C.; Ciccioli, A.; Gigli, G.; Latini, A. On the Thermal and Thermodynamic (In)Stability of Methylammonium Lead Halide Perovskites. Sci. Rep. 2016, 6, No. 31896.

(25) Eperon, G. E.; Stranks, S. D.; Menelaou, C.; Johnston, M. B.; Herz, L. M.; Snaith, H. J. Formamidinium lead trihalide: a broadly tunable perovskite for efficient planar heterojunction solar cells. Energy Environ. Sci. 2014, 7, 982-988.

(26) Saidaminov, M. I.; Abdelhady, A. L.; Maculan, G.; Bakr, O. M. Retrograde solubility of formamidinium and methylammonium lead halide perovskites enabling rapid single crystal growth. Chem. Commun. 2015, 51, 17658-17661.

(27) Binek, A.; Hanusch, F. C.; Docampo, P.; Bein, T. Stabilization of the Trigonal High-Temperature Phase of Formamidinium Lead Iodide. J. Phys. Chem. Lett. 2015, 6, 1249-1253.

(28) Ning, Z.; Dong, H.; Zhang, Q.; Voznyy, O.; Sargent, E. H. Solar cells based on inks of n-type colloidal quantum dots. ACS Nano 2014, 8, 10321-10327.

(29) Xu, F.; Gerlein, F.; Ma, X.; Haughn, C.; Doty, M.; Cloutier, S. Impact of Different Surface Ligands on the Optical Properties of $\mathrm{PbS}$ Quantum Dot Solids. Materials 2015, 8, 1858-1870.

(30) Choi, J. J.; Luria, J.; Hyun, B.-R.; Bartnik, A. C.; Sun, L.; Lim, Y.-F.; Marohn, J. A.; Wise, F. W.; Hanrath, T. Photogenerated Exciton Dissociation in Highly Coupled Lead Salt Nanocrystal Assemblies. Nano Lett. 2010, 10, 1805-1811.

(31) Luther, J. M.; Law, M.; Song, Q.; Perkins, C. L.; Beard, M. C.; Nozik, A. J. Structural, Optical, and Electrical Properties of SelfAssembled Films of PbSe Nanocrystals Treated with 1,2-Ethanedithiol. ACS Nano 2008, 2, 271-280.

(32) Voznyy, O.; Levina, L.; Fan, F.; Walters, G.; Fan, J. Z.; Kiani, A.; Ip, A. H.; Thon, S. M.; Proppe, A. H.; Liu, M.; Sargent, E. H. Origins of Stokes Shift in PbS Nanocrystals. Nano Lett. 2017, 17, 7191-7195.

(33) Luque, A.; Martí, A.; López, N.; Antolín, E.; Cánovas, E.; Stanley, C.; Farmer, C.; Díaz, P. Operation of the intermediate band solar cell under nonideal space charge region conditions and half filling of the intermediate band. J. Appl. Phys. 2006, 99, No. 094503.

(34) Sugaya, T.; Amano, T.; Mori, M.; Niki, S. Miniband formation in InGaAs quantum dot superlattice. Appl. Phys. Lett. 2010, 97, No. 043112.

(35) Sykora, M.; Koposov, A. Y.; McGuire, J. A.; Schulze, R. K.; Tretiak, O.; Pietryga, J. M.; Klimov, V. I. Effect of Air Exposure on Surface Properties, Electronic Structure, and Carrier Relaxation in PbSe Nanocrystals. ACS Nano 2010, 4, 2021-2034.

(36) Kumar, T.; Prabhakar, S.; Raju, G. Adsorption of Oleic Acid at Sillimanite/Water Interface. J. Colloid Interface Sci. 2002, 247, 275281.

(37) Hills-Kimball, K.; Nagaoka, Y.; Cao, C.; Chaykovsky, E.; Chen, $O$. Synthesis of formamidinium lead halide perovskite nanocrystals through solid-liquid-solid cation exchange. J. Mater. Chem. C 2017, 5, $5680-5684$.

(38) Zhou, Z.; Pang, S.; Ji, F.; Zhang, B.; Cui, G. The fabrication of formamidinium lead iodide perovskite thin films via organic cation exchange. Chem. Commun. 2016, 52, 3828-3831.

(39) Laajalehto, K.; Nowak, P.; Pomianowski, A.; Suoninen, E. Xanthate adsorption at $\mathrm{PbS} /$ aqueous interfaces: Comparison of XPS, infrared and electrochemical results. Colloids Surf. 1991, 57, 319-333.

(40) Chen, S.; Solanki, A.; Pan, J.; Sum, T. C. Compositional and Morphological Changes in Water-Induced Early-Stage Degradation in Lead Halide Perovskites. Coatings 2019, 9, 535-545.

(41) Hinnen, C.; van Huong, C. N.; Marcus, P. A comparative X-ray photoemission study of $\mathrm{Bi} 2 \mathrm{Sr} 2 \mathrm{CaCu} 2 \mathrm{O} 8+$ and $\mathrm{Bi} 1.6 \mathrm{~Pb} 0.4 \mathrm{Sr} 2 \mathrm{Ca}-$ Cu2O8+. J. Electron Spectrosc. Relat. Phenom. 1995, 73, 293-304.

(42) Brown, P. R.; Kim, D.; Lunt, R. R.; Zhao, N.; Bawendi, M. G.; Grossman, J. C.; Bulović, V. Energy Level Modification in Lead Sulfide Quantum Dot Thin Films through Ligand Exchange. ACS Nano 2014, 8, 5863-5872. 
(43) Noda, Y.; Ohba, S.; Sato, S.; Saito, Y. Charge distribution and atomic thermal vibration in lead chalcogenide crystals. Acta Crystallogr., Sect. B 1983, 39, 312-317.

(44) Balazs, D. M.; Dirin, D. N.; Fang, H.-H.; Protesescu, L.; ten Brink, G. H.; Kooi, B. J.; Kovalenko, M. V.; Loi, M. A. CounterionMediated Ligand Exchange for PbS Colloidal Quantum Dot Superlattices. ACS Nano 2015, 9, 11951-11959.

(45) Zherebetskyy, D.; Scheele, M.; Zhang, Y.; Bronstein, N.; Thompson, C.; Britt, D.; Salmeron, M.; Alivisatos, P.; Wang, L.-W. Hydroxylation of the surface of $\mathrm{PbS}$ nanocrystals passivated with oleic acid. Science 2014, 344, 1380-1384.

(46) Sun, Z.; Liu, Z.; Li, J.; Tai, G.-a.; Lau, S.-P.; Yan, F. Infrared Photodetectors Based on CVD-Grown Graphene and PbS Quantum Dots with Ultrahigh Responsivity. Adv. Mater. 2012, 24, 5878-5883.

(47) Tong, L.; Qiu, F.; Zeng, T.; Long, J.; Yang, J.; Wang, R.; Zhang, J.; Wang, C.; Sun, T.; Yang, Y. Recent progress in the preparation and application of quantum dots/graphene composite materials. RSC Adv. 2017, 7, 47999-48018.

(48) Fan, Y.; Meng, H.; Wang, L.; Pang, S. Review of Stability Enhancement for Formamidinium-Based Perovskites. Sol. RRL 2019, 3, No. 1900215.

(49) Debellis, D.; Gigli, G.; Ten Brinck, S.; Infante, I.; Giansante, C. Quantum-Confined and Enhanced Optical Absorption of Colloidal $\mathrm{PbS}$ Quantum Dots at Wavelengths with Expected Bulk Behavior. Nano Lett. 2017, 17, 1248-1254. 